\title{
Current status and future prospects of single-crystal neutron diffractometer iBIX
}

\author{
Katsuhiro Kusaka ${ }^{1}$, Taro Yamada ${ }^{1}$, Naomine Yano ${ }^{1}$, Takaaki Hosoya ${ }^{1}$, Takashi Ohhara ${ }^{2}$, Ichiro Tanaka ${ }^{1}$, Masaki KATAGIRI ${ }^{1}$ \\ ${ }^{1}$ IFRC, Ibaraki University, Tokai, Japan, 2J-PARC Center, JAEA, TOKAI, Japan \\ E-mail: katsuhiro.kusaka.1129@vc.ibaraki.ac.jp
}

Single crystal neutron diffraction is one of the powerful methods to obtain the structure information including the hydrogen atoms. IBARAKI biological crystal diffractometer called iBIX is a high performance time-of-flight single crystal neutron diffractometer to elucidate the hydrogen, protonation and hydration structures of organic compound and biological macromolecules in various life processes.

iBIX is installed on the beam line No. 3 in Material and Life Science Facility, J-PARC. To realize high performance, we have succeeded to develop a new photon-counting two-dimensional detector system using scintillator sheets and wavelengthshifting fiber arrays for the $X / Y$ axes. Since the end of 2008, iBIX has been available to user experiments supported by Ibaraki University. In 2012, we have upgraded the 14 existing detectors and install the 16 new detectors for diffractometer of iBIX. The total solid angle of detectors subtended by a sample and the average of detector efficiency become 2 and 3 times, respectively [1]. The total measurement efficiency of the present diffractometer was on one order of magnitude from the previous one coupled with the increasing of accelerator power. In the end of 2012, iBIX could be started to user experiments for biological macromolecules in earnest. In 2015, the accelerator power of J-PARC become 400 600kW. Achievement for full data set of biological macromolecules for neutron structure analysis by using iBIX is as follow. Maximum unit cell size was $132 \times 132 \times 132$ angstrom in cube. Average sample volume was about $2 \sim 3 \mathrm{~mm}$ in cube, Average measurement time was about 7 10 days. At the beginning of 2015, we distributed two press releases on scientific outcomes which make the most of the merit of the neutron diffraction experiment by iBIX [2]. Some interesting results obtained by iBIX will be reported in the presentation.

In order to improve the quality of integrated intensity of weak reflections, we developed a profile fitting method for the peak integration of the data reduction software STARGazer. The profile fitting component was applied to the TOF diffraction data set of standard protein samples obtained by iBIX. From the results, the integrated intensities and model structure obtained by the profile fitting method were more accurate than those of summation integration method especially at higher resolution shells [3]. We already prepared its user manual and distribution package of the data reduction software including the profile fitting component.

In the future, the accelerator power of J-PARC will be improved at $1 \mathrm{MW}$. iBIX should be available regularly for full data set measurement of sample size of $1 \mathrm{~mm} 3$. We will continue to develop the data reduction software and beam line instruments in order to improve the accuracy of intensity data obtained from small samples. We should successfully complete to develop the utility equipments for sample environments (heating, extension system for polymer sample, pulse laser system and cooling system for capillary enclosed sample). And then, those equipments will be started to apply for user experiment in 2017. We will report also the development status of iBIX in the presentation.

[1] Kusaka, K. et al. (2013) J. Synchrotron Rad. 20, 994-998

[2] Nakamura,A. et al. (2015) Science Advances e1500263

[3] Yano, N. et al. (2016) Scientific report 6, 36628

Keywords: Neutron diffraction, Single crystal, Biological macromolecule 